\title{
Wichtige Signifikanztests \\ - Artikel Nr. 11 der Statistik-Serie in der DMW -
}

\section{Common significance tests}

Autoren

Institut

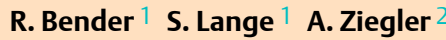

Institut für Qualität und Wirtschaftlichkeit im Gesundheitswesen, Köln

2 Institut für Medizinische Biometrie und Statistik, Universitätsklinikum Schleswig-Holstein,

Campus Lübeck, Universität zu Lübeck
Schlüisselwörter

Signifikanztest

Messniveau

Abhängige Stichproben

Nichtparametrische Methoden

Key words

Significance test

- Measurement scale

Dependent samples

Nonparametric methods

\section{Bibliografie}

DOI 10.1055/s-2007-959034

Dtsch Med Wochenschr 2007;

132: e24-e25 - (c) Georg Thieme Verlag KG Stuttgart · New York . ISSN 0012-0472

Korrespondenz

Privatdozent Dr. rer. biol. hum. Ralf Bender

Institut für Qualität und Wirtschaftlichkeit im Gesundheitswesen (IQWiG)

Dillenburger Straße 27

51105 Köln

eMail Ralf.Bender@iqwig.de
Zum statistischen Nachweis von Unterschieden oder Effekten werden in der medizinischen Forschung häufig Signifikanztests [6] verwendet. Die Grundprinzipien statistischer Tests, die von Lange und Bender [6] am Beispiel des ungepaarten $\boldsymbol{t}$-Tests erläutert werden, gelten auch für andere Testverfahren. Der $\boldsymbol{t}$-Test kommt für viele Testsituationen der medizinischen Forschung in Frage, er deckt aber nicht alle Anwendungsgebiete ab. Je nach Fragestellung und Datensituation benötigt man andere statistische Testmethoden. Die für medizinische Anwendungen wichtigsten statistischen Tests für die einfachen Standardsituationen werden in dieser Arbeit kurz und übersichtlich zusammengefasst.

Zur Auswahl eines adäquaten Tests benötigt man im Wesentlichen die folgenden Informationen:

- die Zahl der zu analysierenden Stichproben

- den Abhängigkeitsstatus der Stichproben

- das Messniveau und die Verteilung der zu analysierenden Zielvariable.

Bei der Stichprobenanzahl genügt die Unterscheidung in 1, 2 oder mehr als 2 Stichproben. Die korrekte Zahl der vorliegenden Stichproben ergibt sich aus der Fragestellung. Möchte man z. B. untersuchen, ob die Erfolgswahrscheinlichkeit einer Behandlungsmethode signifikant über $50 \%$ liegt, so ergibt sich hieraus ein Einstichprobenproblem. Möchte man dagegen die Erfolgswahrscheinlichkeiten zwischen 2 oder mehr als 2 Behandlungsmethoden vergleichen, so liegen 2 bzw. mehr als 2 Stichproben vor.

Der Abhängigkeitsstatus der Stichproben ergibt sich aus dem Studiendesign. Beim Vergleich unverbundener Gruppen liegen unabhängige Stichproben vor. Der Vergleich mehrerer Behandlungsmethoden anhand paralleler Therapiegruppen in randomisierten klinischen Studien und der Vergleich exponierter und nicht exponierter Personen in Kohortenstudien stellen Standardbeispiele für unabhängige Stichproben dar. Beim Vergleich von abhängigen Werten an gleichen oder über gewisse Merkmale verbundenen Un- tersuchungseinheiten liegen abhängige Stichproben vor. Häufige Designs, die zu abhängigen Stichproben führen, sind Crossover-Studien, bei denen alle Patienten die zu vergleichenden Therapien in verschiedenen Studienphasen (meist in zufälliger Reihenfolge) erhalten und gematchte Fall-Kontroll-Studien, bei denen jedem Patient mit der interessierenden Erkrankung („Fälle“) ein oder mehrere Patienten ohne diese Erkrankung („Kontrollen“) so zugeordnet werden, dass Fälle und Kontrollen für bestimmte wichtige Merkmale (z. B. Alter und Geschlecht) gleiche oder zumindest ähnliche Ausprägungen besitzen.

Bezüglich des Messniveaus der Zielvariablen genügt in der Praxis die Unterscheidung zwischen den Messniveaus binär (ja/nein, z. B. Dialysenotwendigkeit), nominal (ungeordnete Kategorien, z. B. Blutgruppe), ordinal (geordnete Kategorien, z. B. Retinopathiestadien), stetig (quantitatives Merkmal, z. B. systolischer Blutdruck) und zensiert (Überlebenszeiten, z. B. Zeit bis zum Tod) [3]. Das Messniveau der Zielvariablen bezieht sich auf die Datenerfassung bei den Untersuchungseinheiten (z. B. Patienten), nicht auf Größen, die aus den Stichprobendaten berechnet werden und diese zusammenfassen. Beispielsweise liegt bei der Untersuchung der Erfolgswahrscheinlichkeit einer Behandlungsmethode in einer definierten Gruppe von Patienten eine binäre Zielvariable vor. Die Erfolgswahrscheinlichkeit ist zwar stetig (zwischen 0 und 1), sie stellt aber eine Größe dar, die aus den einzelnen Stichprobenwerten berechnet wurde; auf der Ebene der Patienten liegt eine binäre Zielvariable vor, nämlich Erfolg ja/nein.

Die üblichen parametrischen Verfahren für stetige Daten setzen strenggenommen auch noch Normalverteilung [1] voraus. Allerdings ist diese Voraussetzung bei großen Stichproben im Allgemeinen vernachlässigbar [8]. Bei kleinen Stichproben mit stetigen aber nicht normalverteilten Daten können die entsprechenden nichtparametrischen Tests, für die mindestens ordinales Messniveau erforderlich ist, verwendet werden. Das Grundprinzip der nichtpa- 
Tab. 1 Übersicht über die wichtigsten statistischen Signifikanztests.

\begin{tabular}{|c|c|c|c|c|c|c|}
\hline \multicolumn{2}{|c|}{ Stichproben } & \multicolumn{2}{|c|}{ Messniveau der Zielvariable } & \multicolumn{3}{|l|}{$t$-Test } \\
\hline Anzahl & Status & binär & nominal & ordinal o. stetig (nicht normal-verteilt) & stetig und normalverteilt & zensiert \\
\hline 1 & - & $\begin{array}{l}\text { Binominaltest } \\
\chi^{2} \text {-Test }\end{array}$ & $\chi^{2}$-Test & $\begin{array}{l}\text { (Vor)zeichentest } \\
\text { Wilcoxon Vorzeichenrangtest }\end{array}$ & $t$-Test & - \\
\hline 2 & unabhängig & $\begin{array}{l}\chi^{2} \text {-Test } \\
\text { Fisher's exakter Test }\end{array}$ & $\begin{array}{l}\chi^{2} \text {-Test } \\
\text { Fisher's exakter Test }\end{array}$ & $\begin{array}{l}\text { Wilcoxon Rangsummentest = } \\
\text { Mann-Whitney U-Test }\end{array}$ & ungepaarter $t$-Test & Log-Rang-Test \\
\hline$>2$ & unabhängig & $\begin{array}{l}\chi^{2} \text {-Test } \\
\text { Fisher's exakter Test }\end{array}$ & $\begin{array}{l}\chi^{2} \text {-Test } \\
\text { Fisher's exakter Test }\end{array}$ & Kruskal-Wallis-Test & F-Test (ANOVA) & Log-Rang-Test \\
\hline 2 & abhängig & McNemar Test & - & $\begin{array}{l}\text { (Vor)zeichentest } \\
\text { Wilcoxon Vorzeichenrangtest }\end{array}$ & gepaarter $t$-Test & - \\
\hline$>2$ & abhängig & Cochran's Q & - & Friedman-Test & $\begin{array}{l}\text { ANOVA für Messwert- } \\
\text { wiederholungen }\end{array}$ & - \\
\hline
\end{tabular}

rametrischen Tests ist die so genannte Rangbildung. In die Berechnung der entsprechenden Teststatistiken gehen nicht die Daten selbst, sondern deren Ränge, d. h. deren Platzierung in der vom kleinsten zum größten Wert sortierten Stichprobe, ein. Ein häufiges Problem in der Praxis stellen hierbei Bindungen dar, d. h. gleiche Ränge durch identische Stichprobenwerte, vor allem bei ordinalen Zielvariablen mit wenigen Kategorien. Eine geringe Anzahl von Bindungen kann in der Praxis vernachlässigt werden. Ist die Anzahl der Bindungen beträchtlich, so sollte man diese berücksichtigen. Meist wird die Bildung von Durchschnittsrängen empfohlen. Für die meisten nichtparametrischen Tests liegt eine so genannte bindungskorrigierte Version vor, die bei großen Stichproben mit vielen Bindungen verwendet werden sollte. Bei kleinen Stichproben $(n<10)$ mit oder ohne Bindungen sollte in jedem Fall die exakte Verteilung der Teststatistik berechnet werden, anstelle der sonst üblichen Approximationen. Die Verwendung exakter Tests ist meist mit einem enormen Rechenaufwand verbunden, allerdings gibt es seit einigen Jahren hierfür spezielle Statistik-Software wie z. B. StatXact [7].

Sowohl die üblichen parametrischen als auch die nichtparametrischen Tests setzen die so genannte Homoskedastizität, d. h. identische Varianzen in den verschiedenen Gruppen, voraus. Diese Annahme ist in der Praxis häufig verletzt. Im Bereich der Therapiestudien findet man z. B. oftmals eine größere Streuung in der behandelten Gruppe, die sich möglicherweise durch ein unterschiedliches Ansprechverhalten auf die Therapie erklären lässt. Ist die Annahme der Homoskedastizität deutlich verletzt, so sollten modifizierte Tests verwendet werden, die keine identischen Varianzen voraussetzen [9]

In Tab. 1 sind die wichtigsten Signifikanztests für die häufigsten und einfachsten Standardsituationen aufgeführt. Es gibt eine Reihe von weiteren Verfahren, die sich wegen ihrer Komplexität nicht in einer einfachen Übersicht zusammenfassen lassen. Ein in der Praxis häufiges Problem ist z. B. der Vergleich unabhängiger Gruppen, wobei nach wichtigen Kovariablen adjustiert werden soll. Hierfür kommen dann als Verallgemeinerung von $\boldsymbol{t}$-Test [6] und Varianzanalyse [2,4] die Methoden der multiplen Regressionsanalyse in Frage [5].

\section{kurzgefasst}

Zur Auswahl eines geeigneten Signifikanztests für die einfachen Standardsituationen in der medizinischen Statistik kann man die Übersicht in Tab. 1 verwenden. Es werden lediglich die Informationen über die Zahl der zu analysierenden Stichproben, deren Abhängigkeitsstatus sowie das Messniveau und die Verteilung der Zielvariable benötigt.
Die englischen Bezeichnungen der hier diskutierten Begriffe zeigt Tab. 2.

Tab. 2 Übersetzung biometrischer Begriffe (deutsch - englisch).

\begin{tabular}{|ll|}
\hline Signifikanztest & significance test \\
\hline Stichprobe & sample \\
\hline unabhängig & independent \\
\hline abhängig & dependent \\
\hline Messniveau & measurement scale \\
\hline binär & binary \\
\hline nominal & nominal \\
\hline ordinal & ordinal \\
\hline stetig & continuous \\
\hline zensiert & censored \\
\hline Überlebenszeit & survival time \\
\hline nichtparametrisch & nonparametric \\
\hline Rang & rank \\
\hline Bindung & tie \\
\hline Kovariable & covariable \\
\hline Vorzeichentest & sign test \\
\hline Wilcoxon Vorzeichenrangtest & Wilcoxon sign rank test \\
\hline Wilcoxon Rangsummentest & Wilcoxon rank sum test \\
\hline geparter t-Test & paired $t$-test \\
\hline Varianzanalyse & analysis of variance (ANOVA) \\
\hline Messwertwiederholungen & repeated measurements \\
\hline Homoskedastizität & homoscedasticity \\
\hline
\end{tabular}

Dieser Beitrag ist eine überarbeitete Fassung aus dem Supplement Statistik aus dem Jahr 2002.

\section{Literatur}

1 Altman DG, Bland JM. The Normal distribution. BMJ 1995; 310: 298

2 Altman DG, Bland JM. Comparing several groups using analysis of variance. BMJ 1996; 312: 1472-1473

3 Altman DG, Bland JM. Time to event (survival) data. BMJ 1998; 317: 468-469

4 Bender R, Ziegler A, Lange S. Varianzanalyse. Dtsch Med Wochenschr 2007; 132: e57-e60

5 Bender R, Ziegler A, Lange S. Multiple Regression. Dtsch Med Wochenschr 2007; 132: e30-e32

6 Lange S, Bender R. Was ist ein Signifikanztest? Dtsch Med Wochenschr 2007; 132: e19-e21

7 Mehta CR, Patel NR. StatXact 5 for Windows. Statistical Software for Exact Nonparametric Inference. CYTEL Software Corporation, Cambridge, MA, 2001

8 Sawilowsky SS, Blair RC. A more realistic look at the robustness and type II error properties of the t test to departures from population normality. Psychol Bull 1992; 111: 352-360

9 Skovlund E, Fenstad GU. Should we always choose a nonparametric test when comparing two apparently nonnormal distributions? J Clin Epidemiol 2001; 54: 86-92 